\title{
High Fill Factor Array of Piezoelectric Micromachined Ultrasonic Transducers with Large Quality Factor
}

\author{
Ding Jiao, ${ }^{1,2}$ Zao Ni, ${ }^{1,2}$ Jiachou Wang, ${ }^{1,2}$ and Xinxin $\mathrm{Li}^{1,2^{*}}$ \\ ${ }^{1}$ State Key Laboratory of Transducer Technology, Shanghai Institute of Microsystem and Information Technology, \\ Chinese Academy of Sciences, 865 Changning Road, Shanghai 200050, China \\ ${ }^{2}$ School of Microelectronics, University of Chinese Academy of Sciences, \\ No. 19(A) Yuquan Road, Beijing 100049, China
}

(Received December 21, 2019; accepted March 18, 2020)

Keywords: PMUT, fill factor, quality factor, acoustic efficiency, aluminum nitride

In this paper, we present a novel piezoelectric micromachined ultrasonic transducer (PMUT) array with a high fill factor and a large quality factor $(Q)$. With the reusable design of etching holes, the pitch between PMUTs is effectively minimized, improving the fill factor of the array. With a newly developed surface micromachining process, the fabricated PMUT array only needs few etching holes to release the structure, and all sealed etching holes are removed from the resonance region, achieving a large $Q$. The demonstrated process facilitates low-cost volume manufacturing. As a result, a $50 \times 50$ PMUT array containing $48-\mu \mathrm{m}$-diameter PMUTs with a $52 \mu \mathrm{m}$ pitch achieves a high fill factor of $67 \%$. For an individual PMUT, a large $Q$ of 351 is achieved at a center frequency of $12.62 \mathrm{MHz}$, the peak displacement is $3.25 \mathrm{~nm} / \mathrm{V}$, and the effective electromechanical coupling coefficient is $1.6 \%$. The achieved high fill factor and large $Q$ render the proposed PMUT array promising in applications such as flow sensing, chemical detection, and energy transmission.

\section{Introduction}

Over the past decade, ultrasonic transducers have been intensively studied and developed for various applications, such as medical imaging, object recognition, flow sensing, and range finding. ${ }^{(1,2)}$ Conventional ultrasonic transducers comprising bulk piezoelectric ceramic materials suffer from acoustic impedance mismatching between the piezoelectric layer and the medium; ${ }^{(3)}$ thus, creating a two-dimensional (2D) matrix ultrasonic array for threedimensional (3D) imaging is difficult. ${ }^{(4)}$ In contrast, micromachined ultrasonic transducers (MUTs) have a compliant membrane structure for good acoustic coupling to the medium, such as air or liquids, ${ }^{(5)}$ and MUTs can incorporate numerous ultrasonic transducers into an array. In addition, MUTs have advantages of miniaturized size, ${ }^{(6)}$ low power consumption, ${ }^{(7)}$ and lowcost volume manufacturing, ${ }^{(8)}$ granting MUTs remarkable potential in consumer electronics and biomedical applications.

Research on MUTs has significantly progressed, and on the basis of the transduction mechanism, they can now be divided into capacitive MUTs (CMUTs) and piezoelectric *Corresponding author: e-mail: xxli@mail.sim.ac.cn https://doi.org/10.18494/SAM.2020.2749 
MUTs (PMUTs). Compared with CMUTs, PMUTs can achieve a desired sensitivity without limitations, such as a high bias voltage ${ }^{(9)}$ or a small gap ${ }^{(10)}$ thus reducing circuit and fabrication complexity. ${ }^{(11)}$ In addition, PMUTs also have a low acoustic impedance and an enhanced sensitivity. ${ }^{(12)}$

However, difficulties in enhancing acoustic efficiency using a low-cost method have prevented the widespread practical application of PMUTs. To increase the acoustic efficiency of PMUTs, the fill factor of the PMUT array should be sufficiently high. On the basis of a typical wet isotropic etching process, various PMUT arrays were fabricated. However, most of these arrays suffer from large PMUT dimensions and pitches resulting from the angled sidewalls during isotropic etching, which generate a low fill factor. Through-wafer deep reactive ion etching (DRIE) is more efficient than wet isotropic etching to improve fill factor as observed in compact PMUT arrays with a PMUT diameter of $50 \mu \mathrm{m}$ and a pitch of $100 \mu \mathrm{m}$ fabricated via DRIE. ${ }^{(13)}$ However, the fill factors of PMUTs are restricted by the aspect ratio of DRIE. To solve the above-mentioned fill factor problem, some PMUT arrays were implemented using silicon-on-insulator (SOI) technology, ${ }^{(14)}$ but the fabrication is costly.

In addition, certain applications, such as flow sensing, chemical detection, and energy transmission, need to use continuous waves excited by PMUTs. ${ }^{(15)}$ Under these circumstances, the quality factor $(Q)$ should be as large as possible to achieve acceptable acoustic efficiency. For example, in a previous work, PMUTs with a sloped profile were fabricated and their $Q$ was improved to $332 ;^{(15)}$ however, the variations of the side wall's inclination are difficult to control.

In this study, we propose a novel PMUT array with improved fill factor and $Q$. By designing a series of reusable etching holes on the surface of the silicon wafer, a compact design of a PMUT array is proposed. The diameter of the proposed PMUT is $48 \mu \mathrm{m}$, and the pitch among the PMUTs is $52 \mu \mathrm{m}$, realizing a fill factor of $67 \%$. By minimizing the number of etching holes and removing them from the resonance region, the $Q$ of an individual PMUT is enhanced to 351. Furthermore, the process presented herein is compatible with the complementary metal oxide semiconductor (CMOS) process, implying that the PMUT array is suitable for highvolume manufacturing with low cost.

\section{Materials and Methods}

\subsection{Design strategy for the PMUT array}

The 3D structure of a $4 \times 4$ PMUT array is presented in Fig. 1(a), with its cross-sectional view shown in Fig. 1(b). The whole transduction structure is integrated on a (100) silicon substrate. The PMUT array demonstrated herein comprises $48-\mu \mathrm{m}$-diameter PMUTs with a 52 $\mu \mathrm{m}$ pitch. The PMUTs are arranged in rows and columns that are electrically connected through the top and bottom electrode metal layers, respectively. Each PMUT in the array is designed to connect with the surrounding etching holes [as shown in Fig. 1(c)] through microetching channels [as shown in Fig. 1(d)] to accelerate the release of the membrane. Furthermore, each etching hole is shared by the surrounding PMUTs, and this design of reusable etching holes minimizes the spacing between PMUTs to $4 \mu \mathrm{m}$, which effectively improves the fill factor of the array. 
(a)
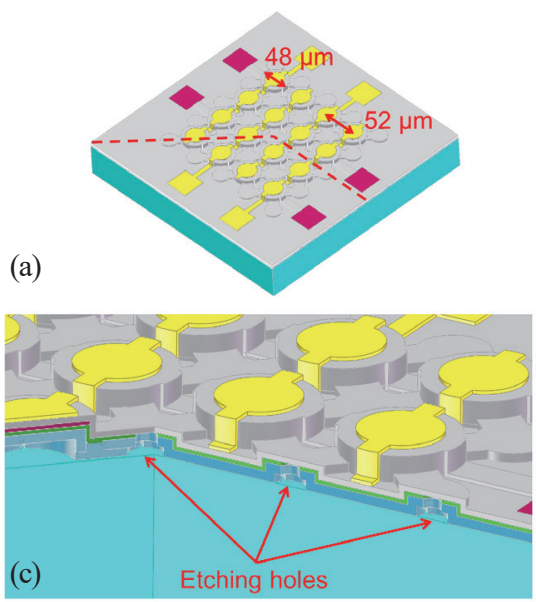
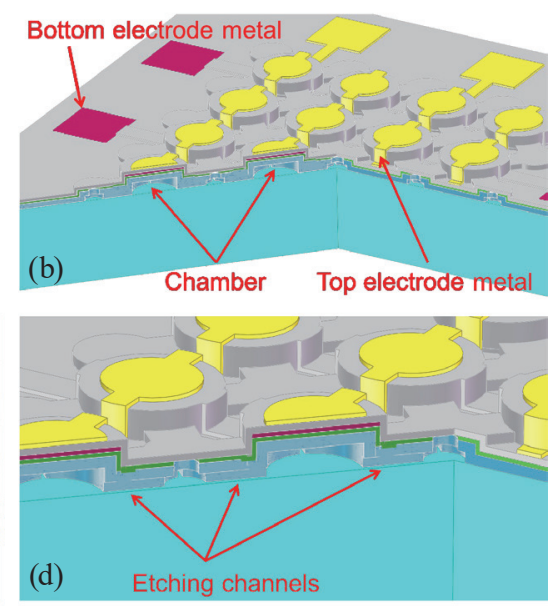

Fig. 1. (Color online) (a) 3D sketch of a $4 \times 4$ PMUT array. (b) 3D cross-sectional view showing the details of the structure, which is cut along the dotted line shown in (a). (c) Magnified view that shows the etching holes. (d) Magnified view that shows the etching channels.

Each PMUT is a piezoelectric unimorph, comprising a piezoelectric layer of aluminum nitride (AlN) covered by $\mathrm{Au}$ and Pt electrodes on the top and bottom, respectively, an insulating layer of $\mathrm{SiO}_{2}$, and an elastic layer of polysilicon. $\mathrm{Cr}$ and $\mathrm{Ti}$ are used as binders for the top and bottom electrodes, respectively. Figure 2 shows the thickness of each layer. Notably, the PMUTs demonstrated herein use AIN as the piezoelectric material because it has a lower dielectric constant than lead zirconate titanate (PZT), which enables high performance, ${ }^{(16)}$ particularly in terms of sensitivity in the receiving mode. Additionally, unlike PZT films, AlN can be deposited at low temperatures $\left(<400{ }^{\circ} \mathrm{C}\right)$ and is fully compatible with CMOS processes. ${ }^{(17)}$

When the voltage signal input from the top and bottom electrodes excites the piezoelectric material, the PMUT vibrates owing to the strain mismatch between the piezoelectric and elastic layers, transmitting ultrasonic waves to the medium. In contrast, when ultrasonic waves act on the piezoelectric material, the surfaces of the piezoelectric material accumulate charges, outputting an electrical signal.

The vibration mode shapes of a PMUT with a pressure of $100 \mathrm{kPa}$ on the upper surface are obtained through the finite element method (FEM), as shown in Fig. 3, wherein the first mode shown in Fig. 3(a) is preferred for the largest displacement, i.e., for a high acoustic coupling efficiency. The first resonant frequency can be calculated using Eq. (1) for a circular PMUT: ${ }^{(18)}$

$$
f_{0}=0.47 \frac{t}{r^{2}} \sqrt{\frac{E}{\rho}},
$$

where $t$ is the thickness of the multilayer, $r$ is the radius of the circular PMUT, $E$ is average Young's modulus, and $\rho$ is the average density of the PMUT. The thicknesses, diameters, 


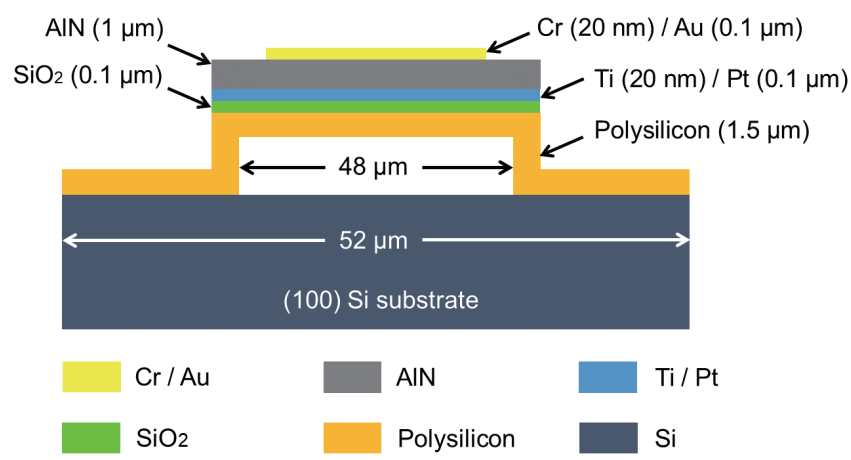

Fig. 2. (Color online) Cross-sectional view of a single PMUT and the thickness of each layer.

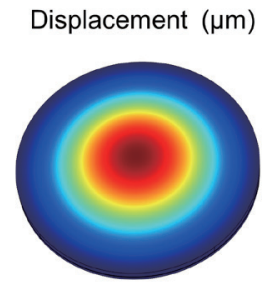

(a)

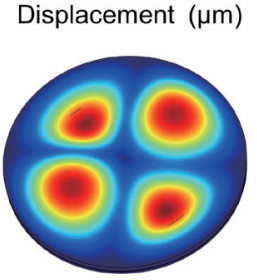

(c)

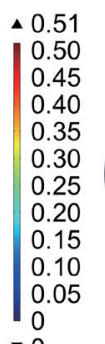

$\checkmark 0$

$\Delta .13$

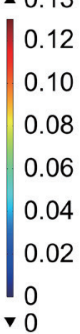

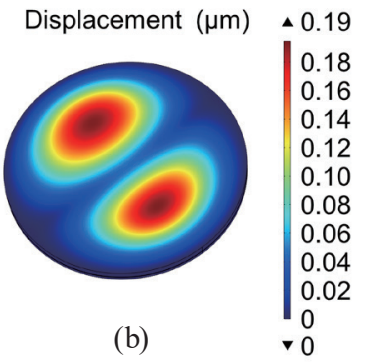

Displacement $(\mu \mathrm{m})$

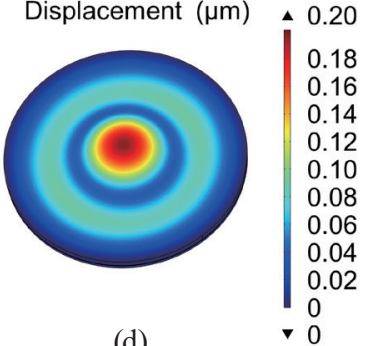

Fig. 3. (Color online) Finite element simulation results of the vibration mode shapes of a circular PMUT. (a)-(d) correspond to the 1 st -4 th vibration mode shapes.

densities, and Young's modules of materials are listed in Table 1. To obtain a resonant frequency above $10 \mathrm{MHz}$, the diameter of an individual PMUT is set as $48 \mu \mathrm{m}$, calculated using Eq. (1). The high resonant frequency of the PMUT provides an adequate axial resolution, and the small pitch of the PMUTs array is desirable for good array performance at high frequencies, such as the increased acoustic efficiency per unit area. Therefore, in the design of the PMUT array, the spacing between the PMUTs has been minimized as far as possible under the fabrication process constraints to achieve a small pitch of $52 \mu \mathrm{m}$.

The electromechanical coupling of PMUTs can be enhanced by optimizing the area of the top electrode. For a circular PMUT with a thin piezoelectric film, the edge of the top electrode should be placed where $\sigma_{r}(d)+\sigma_{\theta}(d)=0$, ${ }^{(14)}$ with $\sigma_{r}$ and $\sigma_{\theta}$ being the stresses in the $r$ and $\theta$ directions, respectively, in polar coordinates in the piezoelectric plane, and $d$ varies from 0 to $48 \mu \mathrm{m}$ along the diameter of the PMUT. By applying a pressure of $100 \mathrm{kPa}$ to the upper surface 
Table 1

Dimensions and material properties used in FEM simulation of PMUT.

\begin{tabular}{lcccc}
\hline Material & Thickness $(\mu \mathrm{m})$ & Diameter $(\mu \mathrm{m})$ & Density $\left(\mathrm{kg} / \mathrm{m}^{3}\right)$ & Young's modulus $(\mathrm{Pa})$ \\
\hline Polysilicon & 1.5 & 48 & 2320 & $160 \times 10^{9}$ \\
$\mathrm{SiO}_{2}$ & 0.1 & 48 & 2200 & $70 \times 10^{9}$ \\
$\mathrm{Ti}$ & 0.02 & 48 & 4506 & $115.7 \times 10^{9}$ \\
$\mathrm{Pt}$ & 0.1 & 48 & 21450 & $168 \times 10^{9}$ \\
$\mathrm{AlN}$ & 1 & 48 & 3300 & $330 \times 10^{9}$ \\
$\mathrm{Cr}$ & 0.02 & 34 & 7150 & $279 \times 10^{9}$ \\
$\mathrm{Au}$ & 0.1 & 34 & 19300 & $70 \times 10^{9}$ \\
\hline
\end{tabular}

of the PMUT, $\sigma_{r}$ and $\sigma_{\theta}$ can be obtained via FEM. On the basis of the results shown in Fig. 4, the diameter of the top electrode was determined to be $34 \mu \mathrm{m}$.

PMUT sensitivity can be improved by optimizing the thickness of the piezoelectric layer. When the applied pressure of $100 \mathrm{kPa}$ is evenly distributed on the upper surface of the PMUT, the voltage at the center of the piezoelectric layer can be obtained via FEM, and this voltage varies as the thickness of the piezoelectric layer changes from 0 to $3 \mu \mathrm{m}$, as shown in Fig. 5 . According to this result, the thickness of the piezoelectric layer is chosen to be $1 \mu \mathrm{m}$ to obtain maximum electrical potential and sensitivity.

Reducing the impact caused by etching holes on the resonant frequency can enhance the $Q$ of the PMUTs. ${ }^{(19)}$ FEM simulation is used to optimize the arrangement of the etching holes. As shown in Fig. 6, the resonant frequency and eigenvalue quality factor are extracted from PMUT models with different numbers of etching holes present around the resonant area. The corresponding result indicates that the PMUT without etching holes has a large eigenvalue quality factor, and the smaller the number of etching holes, the larger the eigenvalue quality factor. Hence, in the PMUTs fabricated herein, four etching holes are set around the resonance region to achieve both a large $Q$ and a high accelerated release rate for the membrane. In addition, keeping the sealed etching holes far from the resonance region can reduce the acoustic wave leakage via the sealed etching holes into the substrate, which helps eliminate anchor loss. ${ }^{(20)}$ Therefore, the etching holes are removed from the resonance region to further increase the $Q$ of the PMUTs.

\subsection{Microfabrication technique}

The PMUT process flow is shown in Fig. 7 and described step-by-step as follows.

(a) The fabrication process begins with a conventional 4-in (100) Si wafer. A 1- $\mu$ m-thick lowtemperature oxide (LTO) layer and a 0.25 - $\mu \mathrm{m}$-thick phosphor silicate glass (PSG) layer are deposited by low-pressure chemical vapor deposition (LPCVD) and patterned in turn. The PSG/LTO layers serve as the sacrificial structures for the PMUT. The LTO layer defines the gap between the function layer and the substrate. The PSG layer covering the LTO layer is used to increase the lateral etching speed, wherein the phosphorous content determines the etching rate. The lateral etching channels only comprise PSG to facilitate sealing in later processes. 


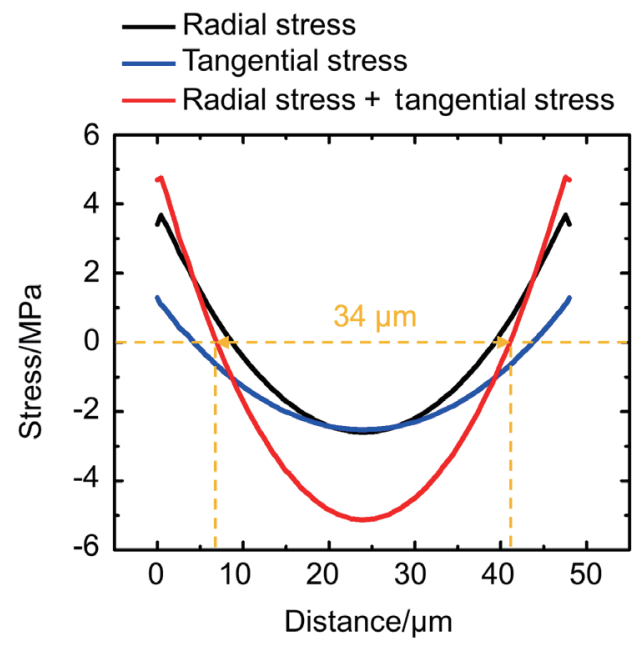

Fig. 4. (Color online) Finite element simulation values of stresses in the $r$ and $\theta$ directions and their sum along a $48-\mu \mathrm{m}$-diameter piezoelectric film with a pressure of $100 \mathrm{kPa}$ applied to the upper surface of the PMUT.

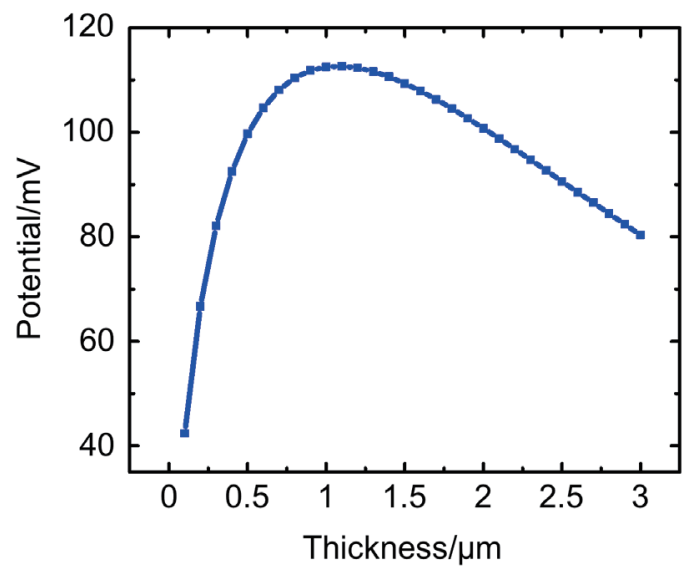

Fig. 5. (Color online) Finite element simulation voltage at the center of the piezoelectric layer with the thickness of the piezoelectric layer varying from 0 to $3 \mu \mathrm{m}$. The applied pressure of $100 \mathrm{kPa}$ is evenly distributed on the upper surface of the PMUT, and the thicknesses of the other layers are shown in Fig. 2.

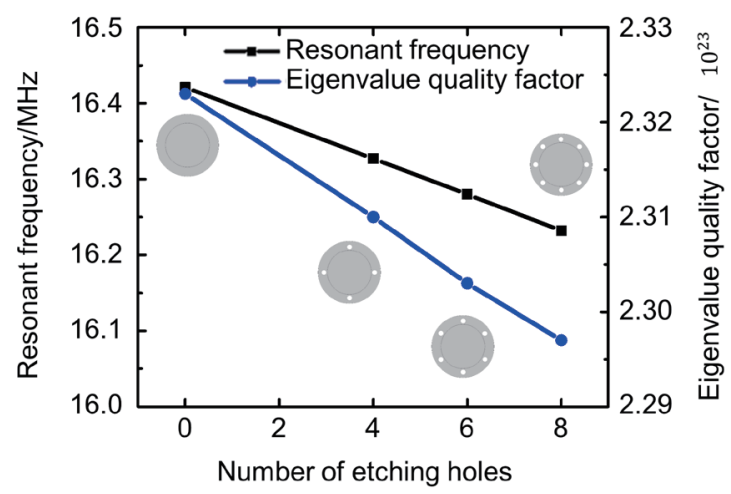

Fig. 6. (Color online) Finite element simulation resonant frequency and eigenvalue quality factor with different numbers of etching holes around the resonant area. 

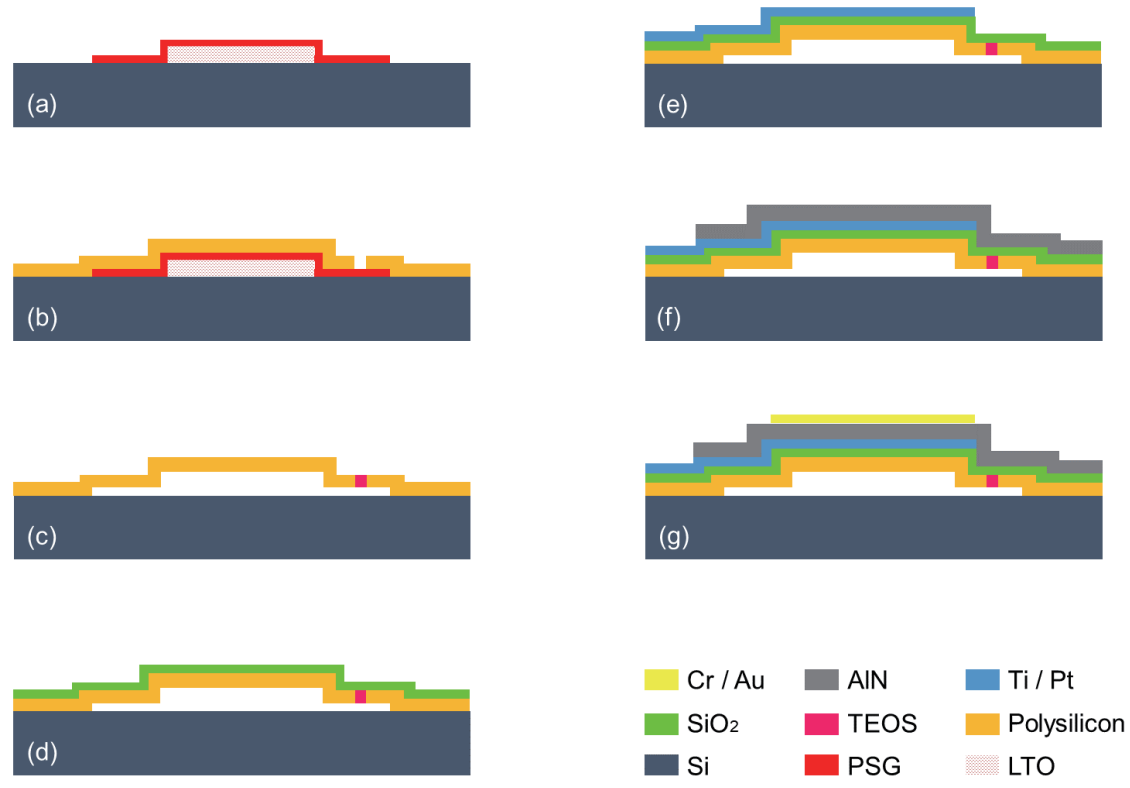

Fig. 7. (Color online) Fabrication of PMUT array.

(b) A $1.5-\mu \mathrm{m}$-thick polysilicon layer is deposited via LPCVD at $620{ }^{\circ} \mathrm{C}$. The polysilicon layer serves as an elastic layer in the PMUT. After the polysilicon layer is annealed to eliminate residual stress, it is patterned via RIE to open the etching holes for sacrificial layer etching.

(c) The sacrificial layer is removed using $40 \% \mathrm{HF}$ for $8-10 \mathrm{~min}$. Then, a $2-\mu \mathrm{m}$-thick tetraethyl orthosilicate (TEOS) oxide layer is deposited via LPCVD and patterned via buffered oxide etching (BOE) to seal the etching holes. The reasons for choosing the TEOS oxide layer as the sealing plug are as follows: (1) the TEOS oxide layer provides excellent conformal coverage to etching holes, and (2) the low vacuum during TEOS oxide layer deposition $\left(50 \mathrm{~Pa}\right.$ at $720^{\circ} \mathrm{C}$ ) simultaneously vacuums the chamber of the PMUT. As the wafer is cooled to room temperature $\left(25^{\circ} \mathrm{C}\right)$, the reference pressure in the chamber of the PMUT further decreases to about $15 \mathrm{~Pa}(100 \mathrm{mTorr})$.

(d) A $0.1-\mu \mathrm{m}$-thick thermal $\mathrm{SiO}_{2}$ layer, which serves as an insulation layer in the PMUT, is grown.

(e) A 20-nm-thick Ti layer and a 0.1- $\mu$ m-thick Pt layer are sputtered in order and patterned via the lift-off technique. The Pt layer serves as the bottom electrode and the Ti layer serves as its binder layer.

(f) A 1- $\mu \mathrm{m}$-thick AlN layer is sputtered and patterned via the iron beam technique to expose the bottom electrode.

(g) Finally, a 20-nm-thick Cr layer and a $0.1-\mu \mathrm{m}$-thick Au layer are sputtered in order and patterned via the lift-off technique. The Au layer serves as the top electrode and the $\mathrm{Cr}$ layer serves as its binder layer.

A scanning electron microscopy (SEM) image of the fabricated $50 \times 50$ PMUT array is shown in Fig. $8(\mathrm{a})$, where the array size is only $3 \times 3 \mathrm{~mm}^{2}$. Each PMUT fabricated herein has a diameter of $48 \mu \mathrm{m}$ with a pitch of $52 \mu \mathrm{m}$, as shown in Fig. 8(b). A comparison of the dimensions and fill factors of the PMUT array is shown in Table 2. The present array achieves 

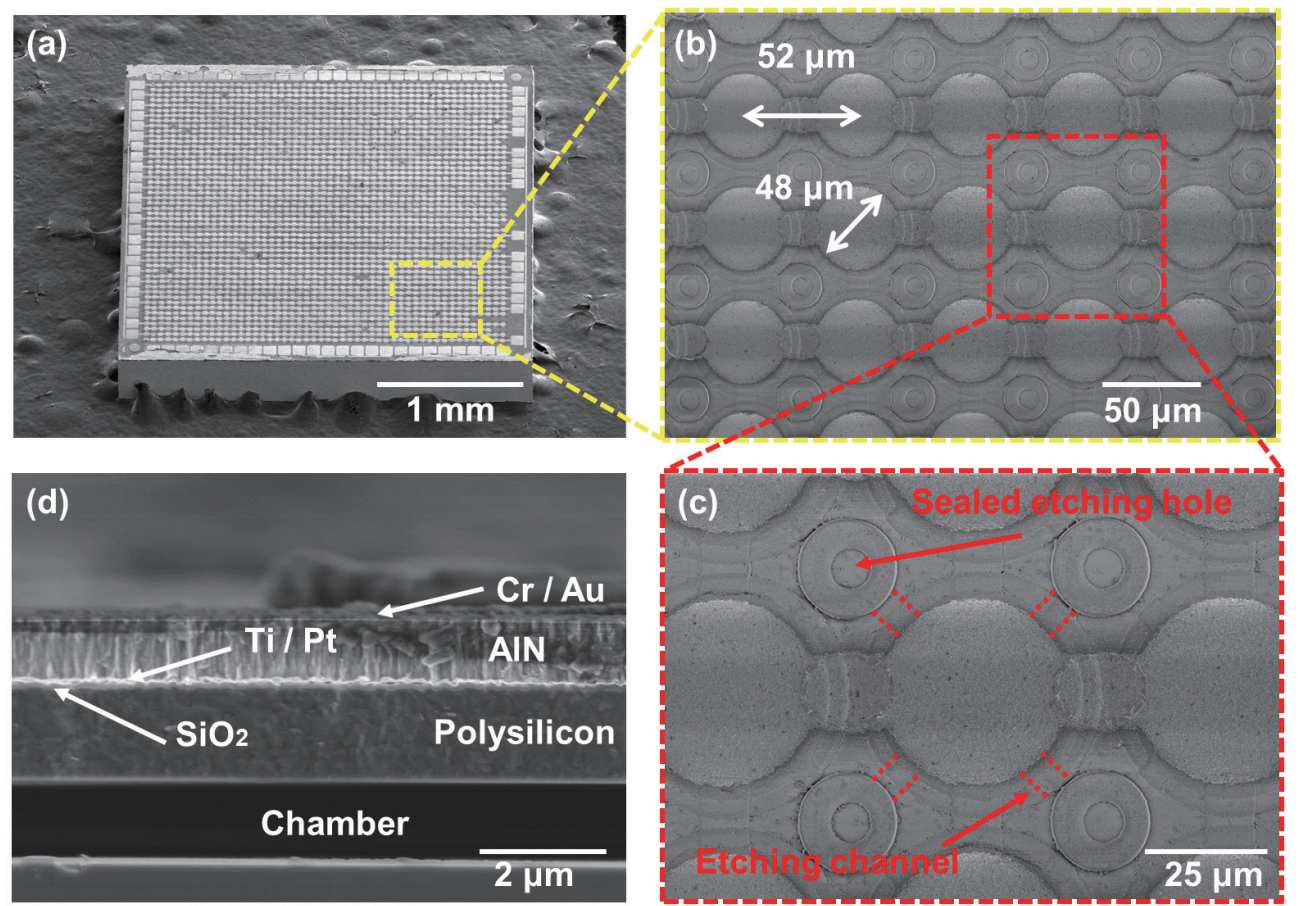

Fig. 8. (Color online) (a) SEM image of the fabricated $50 \times 50$ PMUT array. (b) Top view of the PMUT array. The diameter of a single PMUT is $48 \mu \mathrm{m}$, and the pitch of the PMUT array is $52 \mu \mathrm{m}$, achieving a fill factor of $67 \%$. (c) The microchannels and sealed etching holes can be clearly observed; their compact arrangement realizes the high fill factor. In addition, one etching hole is reused by four surrounding PMUTs, and the etching holes are placed far from the resonance area, achieving a large $Q$. (d) Cross-sectional view of a PMUT. The etching chamber can be clearly observed, with the polysilicon, $\mathrm{SiO}_{2}, \mathrm{Ti} / \mathrm{Pt}, \mathrm{AlN}$, and $\mathrm{Cr} / \mathrm{Au}$ layers above it.

Table 2

Comparison of dimensions and fill factors of PMUT array.

\begin{tabular}{lccc}
\hline PMUT dimension & Pitch $(\mu \mathrm{m})$ & Fill factor $(\%)$ & Reference \\
\hline $50 \times 50 \mu \mathrm{m}^{2}$ & 100 & 25 & Ref. 13 \\
Diameter $=100 \mu \mathrm{m}$ & 150 & 35 & Ref. 21 \\
Diameter $=510 \mu \mathrm{m}$ & 650 & 48 & Ref. 22 \\
$100 \times 125 \mu \mathrm{m}^{2}$ & 150 & 56 & Ref. 23 \\
Diameter $=48 \mu \mathrm{m}$ & 52 & 67 & This work \\
\hline
\end{tabular}

a fill factor of $67 \%$. Compared with those of previously published PMUT arrays, ${ }^{(13,21-23)}$ the fill factor of the PMUT array proposed in this paper is improved by about $20-168 \%$, which is attributed to the reusable design of etching holes in the PMUT array, and the proposed PMUT array features both the very small PMUT size and the very small pitch between PMUTs. The magnified view in Fig. 8(c) shows the microchannels and sealed etching holes, the compact arrangement of which realizes a small space of $4 \mu \mathrm{m}$ between the PMUTs, thus achieving a high fill factor of the PMUT array. Additionally, one etching hole is reused by four surrounding PMUTs to reduce the number of etching holes around the resonance region, and the etching 
holes are placed far from the resonance area, thereby increasing the $Q$ of the PMUTs. The cross-sectional view of a PMUT is shown in Fig. 8(d), wherein the etching chamber, elastic layer of polysilicon, isolated layer of $\mathrm{SiO}_{2}$, bottom electrode of $\mathrm{Pt}$, piezoelectric layer of $\mathrm{AlN}$, and top electrode of Au can be clearly observed.

\section{Results and Discussion}

The X-ray diffraction (XRD) test is implemented to characterize the quality of the AlN film. As observed in Fig. 9, a peak exists near $36.04^{\circ}$, implying that AlN is sputtered onto the substrate along the preferred orientation of 002 , resulting in a good piezoelectric performance of AlN.

A laser dropper vibrometer (LDV, Polytec) is used to measure the displacement frequency response in air. The measurement is first conducted using an $8 \mathrm{~V}$ chirp signal with a frequency ranging between 10 and $15 \mathrm{MHz}$ to excite a single PMUT. As shown in Fig. 10, the PMUT has a large $Q$ of 351 with a center frequency of $12.62 \mathrm{MHz}$. Compared with the previously published PMUT arrays, ${ }^{(13,15)}$ the proposed PMUT array generally exhibits a $Q$ larger than those indicated in Ref. $13(Q=198)$ and Ref. $15(Q=332)$, which is attributed to that few etching holes are needed to release the PMUT structure, and all sealed etching holes are removed from the resonance region in the PMUT array. More importantly, the herein proposed PMUT array features high-yield, low-cost, and volume fabrication with a simple surface micromachining process. Then, the PMUT is excited using an $8 \mathrm{~V}$ sinusoidal signal with a frequency of 12.62 $\mathrm{MHz}$, and the peak displacement is observed to be $3.25 \mathrm{~nm} / \mathrm{V}$, as shown in Fig. 11.

The impedance of the fabricated PMUT is measured using an impedance analyzer (4294A, Agilent). Figure 12 shows the measured impedance curves. The resonant frequency derived from the curves is $12.55 \mathrm{MHz}$, which agrees well with the result of the displacement frequency

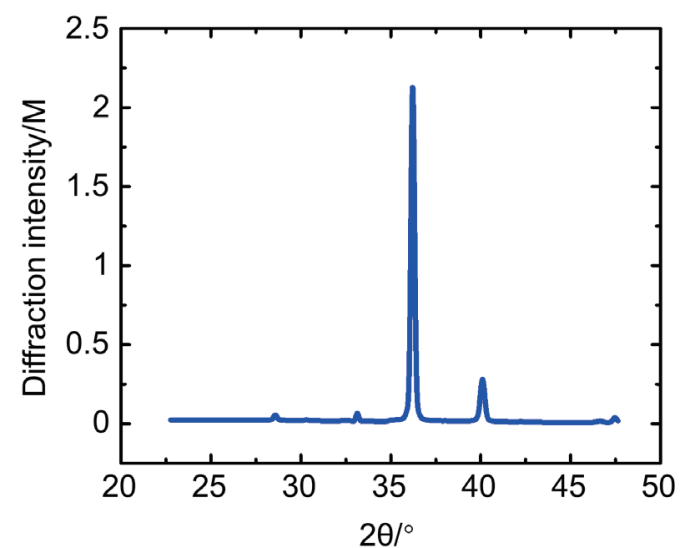

Fig. 9. (Color online) XRD spectrum of the AlN film with a peak near $36.04^{\circ}$.

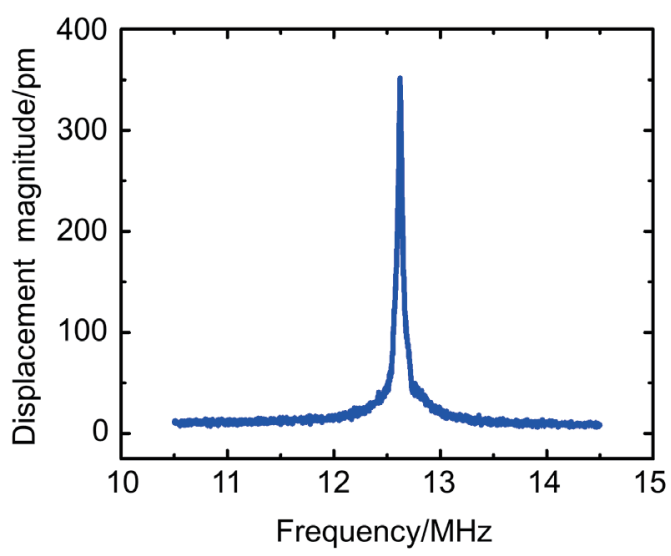

Fig. 10. (Color online) Displacement frequency response in air. The center frequency of the PMUT is $12.62 \mathrm{MHz}$. 


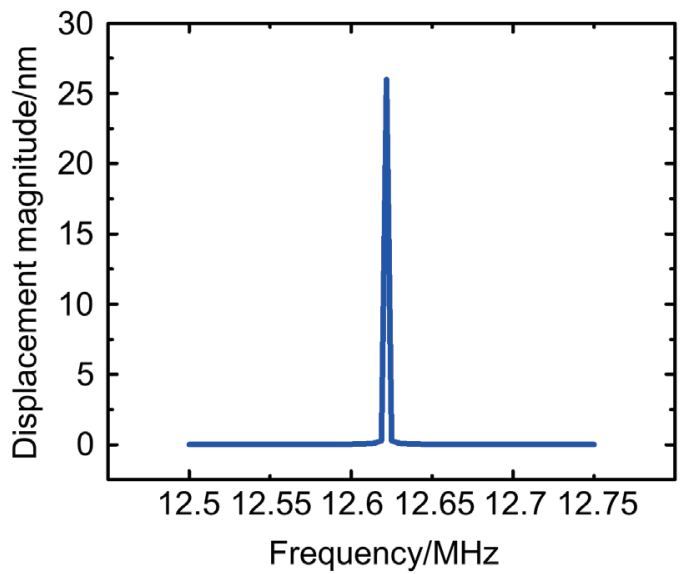

Fig. 11. (Color online) The peak displacement of the PMUT is $3.25 \mathrm{~nm} / \mathrm{V}$.

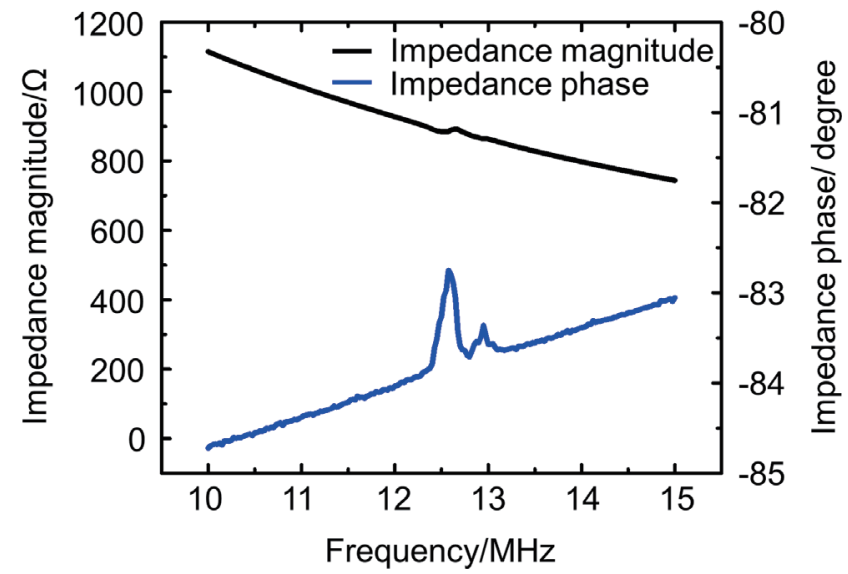

Fig. 12. (Color online) Impedance spectrum of the PMUT. The resonant and antiresonant frequencies derived from the curves were 12.65 and $12.55 \mathrm{MHz}$, respectively.

response, and the antiresonant frequency is $12.65 \mathrm{MHz}$. The effective electromechanical coupling coefficient $k_{\text {eff }}$ is defined as ${ }^{(24)}$

$$
k_{e f f}^{2}=\frac{f_{a}^{2}-f_{r}^{2}}{f_{a}^{2}},
$$

where $f_{a}$ and $f_{r}$ are the antiresonant and resonant frequencies, respectively. On the basis of the $f_{a}$ and $f_{r}$ given above, $k_{\text {eff }}^{2}$ is calculated as $1.6 \%$.

\section{Conclusions}

A PMUT array with a high fill factor and a large $Q$ is proposed herein. The reusable design of etching holes and their optimized arrangement were analyzed and designed to achieve a high fill factor and a high $Q$. The demonstrated process for the present PMUT array is compatible with the CMOS process facilitating low-cost volume manufacturing in standard semiconductor foundries. Results show that the fill factor of the PMUT array and the $Q$ of an individual PMUT are $67 \%$ and 351, respectively, validating the superior performance of the novel transducer design. Compared with those of previously published PMUT arrays,${ }^{(13,21-23)}$ the fill factor of the PMUT array proposed in this paper is improved by about $20-168 \%$, and the PMUT array proposed features both the very small PMUT size and very small pitch between PMUTs. Furthermore, the proposed PMUT array generally exhibits a $Q$ larger than those indicated in Ref. $13(Q=198)$ and Ref. $15(Q=332)$. More importantly, the herein proposed PMUT array features high-yield, low-costs, and volume fabrication with a simple surface micromachining process. The PMUT with a high fill factor and a large $Q$ together with low-cost fabrication is promising for use in applications such as flow sensing, chemical detection, and energy transmission. 


\section{Acknowledgments}

This work was supported in part by the National Key Research and Development Program of China (2016YFA0200803), the Ministry of Science and Technology of China under Grant 2016YFA0200800, and the National Natural Science Foundation of China Projects (61674160, 61834007).

\section{References}

1 B. E. Eovino, Y. Liang, S. Akhbari, and L. Lin: 2018 31st IEEE Int. Conf. Micro Electro Mechanical Systems (MEMS) (IEEE, 2018) 1084. https://doi.org/10.1109/MEMSYS.2018.8346748

2 S. Akhbari, F. Sammoura, B. Eovino, C. Yang, and L. Lin: J. Microelectromech. Syst. 25 (2016) 326. https:// doi.org/10.1109/jmems.2016.2516510

3 Y. Qiu, J. V. Gigliotti, M. Wallace, F. Griggio, C. E. Demore, S. Cochran, and S. Trolier-McKinstry: Sensors 15 (2015) 8020. https://doi.org/10.3390/s150408020

4 Y. Yang, H. Tian, Y. F. Wang, Y. Shu, C. J. Zhou, H. Sun, C. H. Zhang, H. Chen, and T. L. Ren: Sensors 13 (2013) 9624. https://doi.org/10.3390/s130809624

5 S. Shelton, M. Chan, H. Park, D. Horsley, B. Boser, I. Izyumin, R. Przybyla, T. Frey, M. Judy, K. Nunan, F. Sammoura, and K. Yang: 2009 IEEE Int. Ultrason. Symp. (IEEE, 2009) 402. https://doi.org/10.1109/ ULTSYM.2009.5441602

6 T. Wang, T. Kobayashi, and C. Lee: IET Micro Nano Lett. 11 (2016) 558. https://doi.org/10.1049/mnl.2016.0207

7 J. Jung, W. Lee, W. Kang, E. Shin, J. Ryu, and H. Choi: J. Micromech. Microeng. 27 (2017) 113001. https://doi. org/10.1088/1361-6439/aa851b

8 J. Jung, V. Annapureddy, G.-T. Hwang, Y. Song, W. Lee, W. Kang, J. Ryu, and H. Choi: Appl. Phys. Lett. 110 (2017) 212903. https://doi.org/10.1063/1.4983833

9 R. J. Przybyla, S. E. Shelton, A. Guedes, I. I. Izyumin, M. H. Kline, D. A. Horsley, and B. E. Boser: IEEE Sens. J. 11 (2011) 2690. https://doi.org/10.1109/jsen.2011.2157490

10 K. Smyth, S. Bathurst, F. Sammoura, and S. G. Kim: IEEE Trans. Ultrason. Ferroelectr. Freq. Control 60 (2013) 1756. https://doi.org/10.1109/TUFFC.2013.2756

11 Y. Lu, H. Tang, S. Fung, B. E. Boser, and D. A. Horsley: 2015 28th IEEE Int. Conf. Micro Electro Mechanical Systems (MEMS) (IEEE, 2015) 140. https://doi.org/10.1109/MEMSYS.2015.7050905

12 F. Sammoura, K. Smyth, and S. G. Kim: Ultrasonics 53 (2013) 328. https://doi.org/10.1016/j.ultras.2012.06.015

13 Y.-Q. Chen, Y.-X. Li, Y. Chen, Z.-Y. Ju, L.-Q. Tao, Y. Pang, Y. Yang, and T.-l. Ren: J. Electrochem. Soc. 164 (2017) B377. https://doi.org/10.1149/2.0891707jes

14 Y. Lu and D. A. Horsley: J. Microelectromech. Syst. 24 (2015) 1142. https://doi.org/10.1109/ jmems.2014.2387154

15 G. Luo, Q. Wang, Y. Kusano, and D. Horsley: 2018 IEEE Int. Freq. Control Symp. (IFCS) (IEEE, 2018$) 1$. https://doi.org/10.1109/FCS.2018.8597511

16 Y. Lu, A. Heidari, and D. A. Horsley: J. Microelectromech. Syst. 24 (2015) 904. https://doi.org/10.1109/ jmems.2014.2358991

17 E. Yarar, V. Hrkac, C. Zamponi, A. Piorra, L. Kienle, and E. Quandt: AIP Adv. 6 (2016) 075115. https://doi. org $/ 10.1063 / 1.4959895$

18 D. A. Horsley, R. J. Przybyla, M. H. Kline, S. E. Shelton, A. Guedes, O. Izyumin, and B. E. Boser: 2016 IEEE 29th Int. Conf. Micro Electro Mechanical Systems (MEMS) (IEEE, 2016) 145. https://doi.org/10.1109/ MEMSYS.2016.7421579

19 X. Chen, X. Liu, T. Wang, X. Le, F. Ma, C. Lee, and J. Xie: J. Micromech. Microeng. 28 (2018) 057001. https:// doi.org/10.1088/1361-6439/aablbc

20 J. Zou, C.-M. Lin, and A. P. Pisano: 2015 Joint Conf. IEEE Int. Frequency Control Symp. and the European Frequency and Time Forum (IEEE, 2015) 432. https://doi.org/10.1109/fcs.2015.7138874

21 D. Akai, T. Yogi, I. Kamja, Y. Numata, K. Ozaki, K. Sawada, N. Okada, K. Higuchi, and M. Ishida: 2011 16th Int. Solid-State Sens., Actuators Microsyst. Conf. (2011) 910. https://doi.org/10.1109/ TRANSDUCERS.2011.5969159

22 O. Rozen, S. T. Block, X. Mo, W. Bland, P. Hurst, J. M. Tsai, M. Daneman, R. Amirtharajah, and D. A. Horsley: 2016 IEEE 29th Int. Conf. Micro Electro Mechanical Systems (MEMS) (IEEE, 2016) 115. https://doi.org/10.1109/MEMSYS.2016.7421571

23 D. E. Dausch, J. B. Castellucci, D. R. Chou, and O. T. V. Ramm: IEEE Trans. Ultrason. Ferroelectr. Freq. Control 55 (2008) 2484. https://doi.org/10.1109/TUFFC.956

24 J. Jung, S. Kim, W. Lee, and H. Choi: J. Micromech. Microeng. 23 (2013) 125037. https://doi.org/10.1088/0960$1317 / 23 / 12 / 125037$ 